\title{
Getting personal on overdiagnosis: on defining overdiagnosis from the perspective of the individual person
}

Running title: Getting personal on overdiagnosis

Author:

Bjørn Hofmann PhD ${ }^{1,2}$

1. Institute for the Health Sciences at the Norwegian University of Science and Technology (NTNU) at Gjøvik, Norway

2. Centre of Medical Ethics at the University of Oslo, Norway

Corresponding author:

Bjørn Hofmann, The Norwegian University of Science and Technology (NTNU)

PO Box 1

N-2802 Gjøvik, Norway, bjoern.hofmann@ntnu.no

Financial support: There is no external financing of this research.

Word count of manuscript text: 2752

Key Words: overdiagnosis, harm, suffering, disease, definition

Number of references: 33

\section{Acknowledgement}

Part of this work is inspired by stay at the Brocher Foundation outside Geneva April 2018 and the project Conceptual and Ethical Challenges of Overdiagnosis. Although the paper is not part of the project, I am most thankful to the Brocher foundation and to Wendy Rogers, Stacy Carter, and Lynette Reid for highly stimulating and fruitful conversations. 


\title{
Getting personal on overdiagnosis: on defining overdiagnosis from the perspective of the individual person
}

\begin{abstract}
:
How can overdiagnosis be defined, explained, and estimated on an individual level? The answers to this question are essential for persons to be able to make informed choices and give valid consents for tests. Traditional conceptions of overdiagnosis tend to depend on counterfactual thinking and prophetic abilities as you would have to know what would happen in the future if you did not test now. In order to avoid this, overdiagnosis can be defined as the event of diagnosing a person with a disease when this does not avoid or reduce manifest disease. To be able to relate this to one's own life and deliberation, I argue that we need answers to specific questions such as: if I am tested, and the test and subsequent test results are positive, but I am not treated, what is the chance that I would not suffer or experience manifest disease? A definition of overdiagnosis that aims at providing answers to this question is as follows: prospectively overdiagnosis (of an individual person) is the estimated chance that a person having a positive test result would not suffer and experience manifest disease if not treated or followed up in any way. Getting personal on overdiagnosis directs the attention of overdiagnosis estimates towards what matters in medicine: the experience of individual persons.
\end{abstract}




\section{Introduction}

In ordinary language overdiagnosis is defined as «the detection of abnormalities that are not destined to ever bother us ${ }^{1}$ and is identified to entail a series of ethical challenges. ${ }^{2}$ Despite substantial efforts and several explicit attempts ${ }^{3-8}$ there are still disagreements on how to define overdiagnosis ${ }^{9-15}$ and how to measure it. ${ }^{16,17}$ The disagreements with respect to its nature and extension have implications for how to handle overdiagnosis: some think it is a small problem and others think it is substantial; some think it is inevitable while other think it is not.

The conceptual debates are running along several lines. For one, there is disagreement whether overdiagnosis results from biological attributes of the disease, from features of the healthcare system, or from a broader social setting. ${ }^{18,19}$ Secondly, it is disputed what kinds of benefits and harms to include in definitions of overdiagnosis ${ }^{20}$ and who gets to decide ${ }^{2}$. Thirdly, the distinction between overdiagnosis and medicalization is contested, ${ }^{7,12,19}$ and fourthly, there is a debate on whether overdiagnosis hinges on the expansion of disease ${ }^{4,5}$ or on specific diagnostic processes. ${ }^{21,22}$ Fifthly, there are interesting disputes on what types of overdiagnosis that exist, ${ }^{6,20,23}$ whether it is a matter of things in the world (ontology) or of knowledge (epistemology), ${ }^{23}$ and what are its causes. ${ }^{6}$ Last but not least, it is disputed whether overdiagnosis should be defined on the population level, ${ }^{2}$ on the professional level, ${ }^{4,5}$ or on the individual level. ${ }^{6,10,22}$

This article does not pretend to solve all these problems. It will rather focus on a small, but very significant, question: how can overdiagnosis be defined, explained, and estimated on an individual level? The reason this is a crucial question is because in order for persons invited to take tests to be able to make informed choices and give valid consents they should know both the potential benefits and harms of a test, including overdiagnosis. To address this issue, I will refer to some of the definitions that exist, showing how they depend on counterfactual thinking and prophetic abilities. Then I will work my way to a definition of overdiagnosis that tries to avoid these challenges. By investigating questions that individual persons should be provided answers to when being offered a test I will arrive at an operational definition of overdiagnosis targeted for individual persons.

\section{Overdiagnosis of a person}

Although overdiagnosis is frequently defined ${ }^{2}$ and estimated ${ }^{16}$ on the populational level, some of its definitions refer to individuals. Zackrisson defines overdiagnosis as "the detection of cases that would never have come to clinical attention without screening" ${ }^{24}$ and Welch and colleagues at Dartmouth College explain that "[o]verdiagnosis occurs when individuals are diagnosed with conditions that will never cause symptoms or death". ${ }^{1}$ Correspondingly, Etzoni defines overdiagnosis as the "detection of a disease that in the absence of screening would not have been diagnosed in the patient's lifetime." ${ }^{25}$ and Reich as "disease that would never have become clinically evident, a pathological finding lacking clinical import, detectable only by special means (screening or autopsy)."26

Common to all these definitions is that they are based on counterfactual reasoning and presuppose prophetic ability: they presume that you know what would have happened in the future if you did 
something else than what you are doing, i.e., testing and diagnosing (and treating) the person with respect to a specific disease ${ }^{6}$. This makes them incomprehensible for persons and difficult to apply by health professionals when explaining overdiagnosis to persons offered tests.

\section{Defining overdiagnosis}

Nonetheless, let us start with a tentative definition: Overdiagnosis (on an individual person level) is when a person is given a diagnosis (based on one or subsequent tests) but where the person would not have suffered manifest disease if not tested. Here manifest disease means that my suffering (illness) can be explained by palpable or verified signs interpreted by professionals (disease), and accepted by society as sufficient for attributing a sick role (sickness). ${ }^{27}$ As such, overdiagnosis is an unnecessary diagnosis (followed by a potentially unnecessary treatment) in terms of avoiding or reducing a person's future manifest disease. As this definition is in line with traditional definitions of overdiagnosis. ${ }^{1,24-26,28,29}$ it is counterfactual, i.e., you would have to know what would have happened if you did not discover what you have discovered, and you would need to be prophetic to know whether a person will be overdiagnosed or not. ${ }^{6}$

A more prospective definition could be: Overdiagnosis (of an individual person) is when the person is given a diagnosis (based on one or subsequent tests) but who will not avoid suffering manifest disease as a result of the test and subsequent treatment. Some would argue that one problem with this definition is that it includes persons who are correctly diagnosed with the conditions, but who despite early detection and treatment would still suffer manifest disease, i.e., where (early) detection has no effect. There may not be many such cases, but one could argue that the definition is too inclusive, as it comprises cases that are "correctly diagnosed" but where the ultimate effect of the early diagnosis is none or poor. This would make the definition of overdiagnosis dependant on treatment (effects). For some, this is fully acceptable, as they think that non-actionable diagnoses are futile, and because there are many examples of conditions being given diagnose names and handled as diseases because they are treatable. However, in a more principled and epistemic perspective it is fair to argue that if overdiagnosis is about diagnosis, the effects of treatment should be excluded from its definition.

A modified definition that may avoid this problem of treatment-inclusivity would be the following: Overdiagnosis (of an individual person) is when the person is given a diagnosis (based on one or subsequent tests), and does not suffer or experience the manifest disease when left untreated (for the rest of the person's lifetime). Although this definition does not presuppose prophetic abilities, you will still need to know what happens if the person tested (positive) and would not be followed up. To wait and see whether the person developed manifest disease without any intervention when having a positive test, would give an answer to whether the person is overdiagnosed, but that would not be an attractive option to many due to the risk of developing manifest disease. No doubt, it would be possible to design studies that would comply with the above definition and give valuable information on overdiagnosis. However, it would be difficult to have such studies approved by ethical committees and to recruit participants. Nonetheless, the definition is not utopian, as various kinds of

\footnotetext{
'Some would prefer "not maintaining or improving the person's health" instead of "avoiding or reducing a person's future manifest disease".
} 
(bio)markers may emerge in the future that could verify overdiagnosis in previous practices." Moreover, this is in line with established thinking about overdiagnosis: "the only way to be certain that an individual has been overdiagnosed is when that individual is never treated, never develops symptoms of cancer, and ultimately dies of something else." ${ }^{1}$

A shorter, but less precise version of this definition goes like this: Overdiagnosis occurs when diagnosing a person does not avoid or reduce manifest disease. This version is somewhat more imprecise as it can be interpreted to include persons who are correctly diagnosed with the condition, but who despite early detection and treatment still would suffer manifest disease (because poor outcome of the treatment). However, a more friendly interpretation of this definition is that it refers to whether it is the diagnosing (and not treatment deficiencies) that results in not avoiding and reducing manifest disease.

Although such definitions may give a better intuitive understanding of what overdiagnosis is about, it is still very difficult to understand what this means for me as a person offered a test. Hence, in order to provide an operational definition that people can relate to and that can be estimated, we should look at what people offered tests might want to know.

\section{What I need to know in order to be able to make informed decisions}

What I need to know is what it means for me to be tested. Hence, providers of tests should be able to provide me with answers to the following questions:

1. What is the chance that I will suffer and experience (this specific) manifest disease in the first place? (That is, what is my background chance of experiencing manifest disease?)

2. If I am tested, and the test is positive, what is the chance that I will suffer and experience manifest disease (if I am not subsequently tested and treated) compared to if I am not tested at all? If there are several steps in being tested, what is chance for each step? (That is, what is my chance of experiencing manifest disease for each step of the testing process?)

3. If I am tested, and the test is positive and if I am subsequently tested and treated, what is the chance that I will avoid suffering and experiencing manifest disease compared to if I am not tested?

4. If I am tested, and the test and subsequent test results are positive, but I am not treated, what is the chance that I would not suffer or experience manifest disease?

5. If I am tested and I have a negative test, what is the chance that I will still suffer and experience manifest disease? If there are several steps before I have a negative test result, what are the chances for each step?

Question 4 provides a practical answer to what overdiagnosis means to me in the situation of being offered a test. Accordingly, the definition of overdiagnosis becomes: Prospectively, overdiagnosis (of an individual person) is the estimated chance that a person having a positive test result would not suffer and experience manifest disease if not treated or followed up in any way.

\footnotetext{
ii It is interesting to note that such biomarkers then at the same time as revealing overdiagnosis in previous practices would eliminate this specific type of overdiagnosis in present practices. New kinds of overdiagnosis (of such biomarkers) may of course emerge.
} 


\section{Discussion}

Starting out asking how overdiagnosis can be defined, explained, and estimated on an individual level, I referred to some of the definitions that exist and showed how they depend on counterfactual thinking and prophetic abilities, which is making it difficult for people to understand, decide, and to consent. Trying to avoid these challenges I worked my way to a tentative definition of overdiagnosis on an individual level. Furthermore, by investigating questions that persons should be provided answers to when being offered a test, I arrived at an operational definition of overdiagnosis targeted for individual persons. However, where does this leave us? I have given definitions of overdiagnosis that explain what it is, but what about providing individuals with relevant estimates? How do we find the estimated chance that a person having a positive test result would not suffer and experience manifest disease if not treated or followed up in any way?

As we cannot provide accurate answers to individual persons for questions Q1-5, we are lead back to the controversies of estimating overdiagnosis..$^{16,17,30-32}$ Nonetheless, the above questions and suggested definition directs the attention and activities directly towards the key stakeholder: the person offered a test. Hence, estimates of overdiagnosis that do not answer question Q4 (and do not fall under the definition above) are not proper estimates of overdiagnosis. One may of course apply other estimates for making health policy decisions, e.g., with respect to whether to offer a screening program or not. However, when such a program is offered, persons need to be informed and answers to Q4 have to be provided. If not, the person cannot make informed choices and give valid consent for being tested. Moreover, it is important to acknowledge that such estimates have to be presented and balanced against estimates of other outcomes of testing, such as those addressed in Q2-5.

Being told that if I am tested, and the test is positive, there is a $15 \%$ chance that I would still not suffer or experience manifest disease if nothing was done (Q4), may be helpful for my deliberation. I could then compare this with the information that if I am tested, and the test is positive and if I am subsequently tested and treated, then the chance that I will avoid suffering and experiencing manifest disease is $20 \%$ (Q3). Depending on information about how often cases are missed (Q5), my chance of experiencing the manifest disease (Q1) for each step of the test trajectory (Q2), the severity of the disease, the effectiveness and the safety of the treatment and other issues, I could make an informed choice whether I should take the test (or participate in the screening program), and I would be enabled to give a valid consent.

The provided definition does not solve the problem of risk perception. ${ }^{33,34}$ Understanding what a given number of a chance for a given outcome (in percent) means for me as a person is, no doubt, challenging. However, being given such a number from the perspective of what matters for me as an individual person is better than being given numbers that matter for decision makers and health professionals. Hence, this is just a first, but important step, to improve decision making on overdiagnosis on the individual person level.

It is also important to notice that there is nothing magical about overdiagnosis and its estimates. Medicine is quite used to counterfactual and "prophetic" thinking. The prognosis of a disease is exactly such a prophecy, and health professionals are used to think about and estimate prognostic measures. They are also used to think about and explain to patients the prospective of alternative treatments (including non-treatment). "In this situation you can choose between treatment X and 
treatment $Y$. Based on population studies the outcomes of $X$ and $Y$ are $O_{x}$ and $O_{y}$, and the chances of obtaining such outcomes are $C_{x}$ and $C_{y} . "$

It may be argued that overdiagnosis does not have to be defined in terms of manifest disease defined in terms of personal, professional, and social perspectives on human malady (illness, disease, sickness). I have addressed this issue in another paper that points out that the source of the moral imperative to help people is experienced and manifest disease (submitted manuscript).

In order to decide whether I will have a test or not or whether I will enter a screening program or not, I need information that makes it possible to understand what it means to me. Hence, very stringent population based estimates of overall overdiagnosis rate from RCTs may not provide me with the best information. Information from specific observational studies may be more appropriate. Hence, getting personal on overdiagnosis also challenges traditional epistemic hierarchies in Evidence Based Medicine.

Moreover, based on decision-analytic approaches, it has been suggested to find the minimum acceptable trade-off between the benefit of increased test-positives (TP) and harm of increased false positives (FP) in order to finding the acceptable level of tests. Accordingly, it has been suggested to ask: "What is the highest number of individuals undergoing unnecessary further tests or treatment due to FP you would be prepared to accept for the benefit of identifying one additional TP result?" 35 In this article, I have tried to argue for moving this question from the professional to the person; from the question "how good is the test?" to "what is it good for?" keeping the answer to the question "who is it good for?" fixed.

Yet another objection may be that the provided definition of overdiagnosis does not avoid counterfactuals or prophetic abilities, as the questions are based on conditionals ("if, then...") which presuppose what I try to avoid. iii First, it can be argued that counterfactuals can be seen as a specific type of conditionals (sine qua non) ${ }^{36}$ and that this is neither the case in the "ifs" of the questions nor of the definition. Second, it can be pointed out that that the "ifs" in the questions and in the definition describe what is happening: "if I am tested" or "if not treated or followed up in any way." Third, it can be argued that the "ifs" refer to alternative decisions in a decision tree. Surely, you cannot choose all alternatives at the same time. When choosing one, you exclude alternative decisions. However, this is different from knowing what would happen if you did something else than what you are doing. Providing you with estimates of chances for the various alternatives in a decision tree is by no means to provide you with knowledge about what will happen to you in the future. However, at present they are the best measures we have for improving decision making on taking tests.

\section{Conclusion}

The question of how overdiagnosis can be defined, explained, and estimated on an individual level is essential in order for persons to be able to make informed choices and give valid consents to being tested. Traditional conceptions of overdiagnosis tend to depend on counterfactual thinking and prophetic abilities. Trying to avoid this, I have defined overdiagnosis (of an individual person) occurring when the person is given a diagnosis (based on one or subsequent tests), and the person

\footnotetext{
iii I am most thankful to one of the anonymous reviewers for drawing my attention to this objection.
} 
does not suffer or experience the manifest disease when left untreated (for the rest of the person's lifetime). In short, overdiagnosis occurs when diagnosing a person does not avoid or reduce manifest disease. To be able to relate this to one's own life and deliberation, we need answers to questions like Q4: if I am tested, and the test and subsequent test results are positive, but I am not treated, what is the chance that I would not suffer or experience manifest disease? A definition of overdiagnosis that aims at providing answers to this question is that prospectively, overdiagnosis (of an individual person) is the estimated chance that a person having a positive test result would not suffer and experience manifest disease if not treated or followed up in any way. Getting personal on overdiagnosis does not only direct the attention of overdiagnosis estimates towards the perspective of individual persons, but it also challenges traditional epistemic hierarchies in Evidence Based Medicine.

\section{References}

1. Welch HG, Schwartz L, Woloshin S. Overdiagnosed : making people sick in the pursuit of health. Boston, Mass.: Beacon Press; 2011.

2. Carter SM, Degeling C, Doust J, Barratt A. A definition and ethical evaluation of overdiagnosis. Journal of medical ethics. 2016;42:722-724.

3. Carter SM, Rogers W, Heath I, Degeling C, Doust J, Barratt A. The challenge of overdiagnosis begins with its definition. BMJ. 2015;350:h869.

4. Rogers W, Walker M. Précising definitions as a way to combat overdiagnosis. Journal of evaluation in clinical practice. 2018.

5. Walker MJ, Rogers W. Defining disease in the context of overdiagnosis. Medicine, Health Care and Philosophy. 2017;20(2):269-280.

6. Hofmann B. Diagnosing overdiagnosis: conceptual challenges and suggested solutions. European Journal of Epidemiology. 2014;29(9):599-604.

7. Hofmann B. Medicalization and overdiagnosis: different but alike. Medicine, health care, and philosophy. 2016;19(2):253-264.

8. Brodersen J, Schwartz LM, Heneghan C, O'Sullivan JW, Aronson JK, Woloshin S. Overdiagnosis: what it is and what it isn't. Evidence-based medicine. 2018;23(1):1-3.

9. Hofmann B. Defining and evaluating overdiagnosis. Journal of medical ethics. 2016;42(11):715-716.

10. Hofmann B. The overdiagnosis of what? Barring the expansive conception of disease. Konferanse presented at 4th international conference on Preventing Overdiagnosis; 2016-0920 - 2017-09-22, 2016; Barcelona.

11. Hofmann BM. Conceptual overdiagnosis. A comment on Wendy Rogers and Yishai Mintzker's article "Getting clearer on overdiagnosis". Journal of evaluation in clinical practice. 2017;23(5):1118-1119.

12. Hofmann B. Conceptual overdiagnosis. A comment on Wendy Rogers and Yishai Mintzker's article "Getting clearer on overdiagnosis". Journal of evaluation in clinical practice. 2016.

13. Rogers W. Understanding overdiagnosis as a form of medicalisation. 29th EUROPEAN CONFERENCE ON PHILOSOPHY OF MEDICINE AND HEALTH CARE; August 22, 2015; Ghent.

14. Rogers WA, Mintzker Y. Casting the net too wide on overdiagnosis: benefits, burdens and non-harmful disease. Journal of medical ethics. 2016(42):717-719.

15. Morrison M. Overdiagnosis, medicalisation and social justice: commentary on Carter et al (2016) 'A definition and ethical evaluation of overdiagnosis'. Journal of medical ethics. 2016.

16. Ripping TM, ten Haaf K, Verbeek AL, van Ravesteyn NT, Broeders MJ. Quantifying overdiagnosis in cancer screening: A systematic review to evaluate the methodology. JNCl: Journal of the National Cancer Institute. 2017;109(10). 
17. de Gelder R, Heijnsdijk EA, van Ravesteyn NT, Fracheboud J, Draisma G, de Koning HJ. Interpreting overdiagnosis estimates in population-based mammography screening. Epidemiologic reviews. 2011;33(1):111-121.

18. Carter SM, Doust J, Degeling C, Barratt A. A definition and ethical evaluation of overdiagnosis: response to commentaries. Journal of medical ethics. 2016;42(11):722-724.

19. van Dijk W, Faber MJ, Tanke MA, Jeurissen PP, Westert GP. Medicalisation and Overdiagnosis: What Society Does to Medicine. International journal of health policy and management. 2016;5(11):619-622.

20. Marcus PM, Prorok PC, Miller AB, DeVoto EJ, Kramer BS. Conceptualizing overdiagnosis in cancer screening. Journal of the National Cancer Institute. 2015;107(4):djv014.

21. Hofmann B. The overdiagnosis of what? On the relationship between the concepts of overdiagnosis, disease, and diagnosis. Medicine, health care, and philosophy. 2017;20(4):453464.

22. Hofmann B. Overdiagnostic uncertainty. European Journal of Epidemiology. 2017;32(6):533534.

23. Rogers WA, Mintzker Y. Getting clearer on overdiagnosis. Journal of evaluation in clinical practice. 2016;22(4):580-587.

24. Zackrisson S, Andersson I, Janzon L, Manjer J, Garne JP. Rate of over-diagnosis of breast cancer 15 years after end of Malmö mammographic screening trial: follow-up study. Bmj. 2006;332(7543):689-692.

25. Etzioni R, Penson DF, Legler JM, et al. Overdiagnosis due to prostate-specific antigen screening: lessons from U.S. prostate cancer incidence trends. Journal of the National Cancer Institute. 2002;94(13):981-990.

26. Reich J. A critical appraisal of overdiagnosis: estimates of its magnitude and implications for lung cancer screening. Thorax. 2008;63(4):377-383.

27. Hofmann B. Suffering: Harm to Bodies, Minds, and Persons. In: Schramme T, Edwards S, eds. Handbook of the Philosophy of Medicine. Dordrecht: Springer Netherlands; 2017:129-145.

28. Welch HG, Black WC. Overdiagnosis in cancer. Journal of the National Cancer Institute. 2010;102(9):605-613.

29. Moynihan R, Henry D, Moons KG. Using evidence to combat overdiagnosis and overtreatment: evaluating treatments, tests, and disease definitions in the time of too much. PLoS medicine. 2014;11(7):e1001655.

30. Bae JM. Overdiagnosis: epidemiologic concepts and estimation. Epidemiology and health. 2015;37:e2015004.

31. Feig SA. Pitfalls in accurate estimation of overdiagnosis: implications for screening policy and compliance. Breast cancer research : BCR. 2013;15(4):105.

32. Etzioni R, Gulati R, Mallinger L, Mandelblatt J. Influence of study features and methods on overdiagnosis estimates in breast and prostate cancer screening. Annals of internal medicine. 2013;158(11):831-838.

33. Slovic $P$, Peters E. Risk perception and affect. Current directions in psychological science. 2006;15(6):322-325.

34. Gigerenzer G. Calculated risks: How to know when numbers deceive you. Simon and Schuster; 2015.

35. Pepe MS, Feng Z, Janes H, Bossuyt PM, JD. P. Pivotal evaluation of the accuracy of a biomarker used for classification or prediction: standards for study design. J Nat/ Cancer Inst. 2008;100(20):1432-1438.

36. Lewis D. Counterfactuals. John Wiley \& Sons; 2013. 